\title{
Rational route for targeted synthesis of microporous mixed-metal MOFs
}

$\underline{\text { Ghada Ayoub }}^{1}$, Krunoslav Užarević ${ }^{2}$, Ashlee J. Howarth ${ }^{3}$, Luzia S. Germann ${ }^{4}$, Robert E. Dinnebier ${ }^{4}$, Omar K. Farha ${ }^{3}$, Tomislav Friščić ${ }^{1}$ ${ }^{1}$ Chemistry Department, Mc Gill University, Montreal, Canada, ${ }^{2}$ Institute Ruđer Bošković, Bijenička 54, HR-10000, Zagreb, Croatia,

${ }^{3}$ Department of Chemistry, Northwestern University, 2145 Sheridan Rd., Evanston, United States, ${ }^{4}$ Max Planck Institute for SolidState Research, 70569., Stuttgart, Germany

E-mail: ghada.ayoub@mail.mcgill.ca

MOF-74 materials belong to a family of metal-organic frameworks (MOFs) that has been intensely studied due to their distinguished characteristics which include high microporosity, robustness, and presence of open metal sites. The latter widen the applications of MOF-74 materials in diverse fields such as molecular sensing, gas storage, separation, and catalysis.[1]

Introducing heterogeneity into the MOF-74 scaffold is currently emerging as an attractive strategy to expand their applications in catalysis and magnetism. However, conventional solution-based (solvothermal) approaches to mixed-metal MOF-74 synthesis is still a burden to obtain materials with precise stoichiometric composition.[2] We now present a novel, all solid-state synthetic methodology for targeted, efficient and clean synthesis of microporous mixed-metal MOF-74 frameworks with 1:1 ratio of different divalent metal nodes. Our approach is mechanochemical,[3] and has been developed from detailed in situ X-ray diffraction studies of mechanochemical assembly of Zn-MOF-74.[4] The strategy is based on utilizing a specifically prepared coordination polymer as a primary metal precursor that further assembles with a secondary metal source into the three-dimensional MOF-74 structure. This is, to the best of our knowledge, the first general mechanochemical methodology for the targeted synthesis of mixed-metal MOFs. Based on a combination of proton-transfer, and coordination-driven self-assembly during milling, it permits the combination of diverse metal nodes, including: $\mathrm{Zn}$, CoII, NiII, MnII, CuII, Mg and even Ca.

[1] Howarth, A.J. et al. (2016). Nat. Rev. Mater. 15018

[2] Wang, L.J. et al. (2014). Inorg. Chem. 53, 5881-5883

[3] James et al. (2012). Chem. Soc. Rev. 41, 413-447

[4] Julien et al. (2016). Am. Chem. Soc. 138, 2929-2932

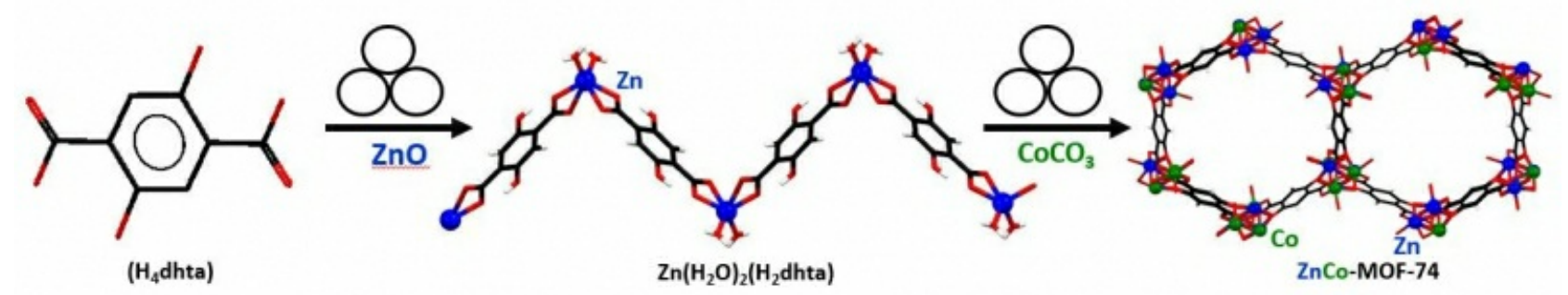

Keywords: mixed-metal MOF-74, mechanochemistry, coordination polymer. 